\title{
Efecto en la gestión organizacional y la satisfacción de los usuarios de un sistema informático de planificación de recursos empresariales (ERP) en Riobamba, Ecuador
}

\author{
Rosa M. Zabala ${ }^{1}$ *, Luis G. Granja ${ }^{2}$, Hugo A. Calderón ${ }^{3}$ y Luis E. Velasteguí ${ }^{4}$ \\ (1) Universidad de Sevilla, Calle San Fernando, 4, 41004, Sevilla, España. (correo-e: roszabcua@alum.us.es) \\ (2) GAD Municipal de Riobamba, 5 de junio y Primera Constituyente, Riobamba-Ecuador, \\ (correo-e: granjal@gadmriobamba.gob.ec) \\ (3) Akzonobel, Mariano Cardenal N73-75 y José Larrea, Quito-Ecuador, hugo.calderonorozco@akzonobel.com \\ (4) Ciencia Digital Editorial, Jardín Ambateño, Ambato-Ecuador, (correo-e: luisefrainvelastegui@cienciadigital.org)
}

Recibido Mar. 1, 2021; Aceptado May. 3, 2021; Versión final Jun. 4, 2021, Publicado Oct. 2021

\section{Resumen}

En el presente estudio se realizó una evaluación ex-post del sistema informático integral (ERP, en inglés) en el gobierno autónomo descentralizado (GAD) municipal del Cantón Riobamba (Ecuador) para conocer el desempeño institucional percibido por los usuarios internos (servidores públicos y directivos) y externos (contribuyentes), e identificar su grado de satisfacción respecto a los servicios municipales. Se utilizaron el modelo de medida multidimensional de DeLone y McLean y métodos estadísticos para el procesamiento y evaluación de los instrumentos aplicados. Los resultados muestran una percepción desfavorable del mejoramiento de la calidad del servicio y la satisfacción de los ciudadanos, así como una relación directa entre las dimensiones calidad y satisfacción del usuario. Se concluye que el sistema ofrece beneficios relacionados con la gestión organizacional, principalmente para los servidores públicos, aunque los directivos, mostraron el mayor nivel de satisfacción como usuarios del sistema.

\section{Enterprise resource planning (ERP) effect on organizational management and user satisfaction in Riobamba, Ecuador}

\begin{abstract}
The primary objective of the present study is to perform an ex-post evaluation of the enterprise resource planning (ERP) integral computer system at the municipal government of Riobamba Canton (Ecuador) to evaluate institutional performance as perceived by internal (public servants and managers) and external (taxpayers) users and assess their satisfaction with municipal services. For this purpose, the DeLone and McLean multidimensional measurement model and statistical methods were used for processing and evaluating the instruments applied. The results showed unfavorable perceptions on service quality improvements and citizen satisfaction. There was also a direct relationship between the dimensions of quality and user satisfaction. It is concluded that the system offers benefits related to organizational management, mainly for public servants, although managers showed the highest level of satisfaction as users of the system.
\end{abstract}




\section{INTRODUCCIÓN}

La corriente del capitalismo advierte hoy transformaciones en el mundo empresarial y gubernamental impulsado por la revolución tecnológica. Diversos autores han enfatizado el papel esencial de la información y el conocimiento como núcleo fundamental del sector industrial y productivo. En este ámbito, las tecnologías de la información y la comunicación conllevan un cambio de paradigma de los procedimientos operativos de relativa importancia para las empresas (Peirano y Suárez, 2006). Con el paso de los años, las organizaciones se han concientizado de los cambios necesarios en sus procesos, estructuras, mercados, tecnologías y demás aspectos de su rol, todo para lograr una mayor competitividad en este mundo globalizado de hoy. Es así que se ha venido aceptando igualmente que, en el proceso dinámico organizacional y su desempeño, la implementación de las Tecnologías de la Información y de la Comunicación juega un papel fundamental en la gestión organizacional ante los cada vez más complejos escenarios. Así, la gestión se ha ampliado a todas las actividades que pueden incidir en los resultados de la organización. Históricamente, en las organizaciones han proliferado múltiples sistemas de gestión, cada uno de ellos encaminado a cumplimentar una serie de actividades de carácter técnico-especializado (Tamayo, 2015).

Las modernas funciones y diferentes procesos en el mundo corporativo se están tornando muy complicadas, principalmente cuando se trata de tomar decisiones muy importantes para las empresas u organizaciones, como por ejemplo establecer los precios más adecuados de sus productos para no perjudicar la demanda del mercado o que no llegue a cubrir mínimamente los costos de producción, o establecer políticas gubernamentales adecuadas que responda a cubrir necesidades ciudadanas en base a factores estadísticos, técnicos, sociales, económicos entre otros (Korolov, 2018).

El uso de las Tecnologías de la Información y de la Comunicación (TIC) está creciendo a un ritmo acelerado y está dando lugar a cambios importantes en la sociedad, permitiendo que la información esté al alcance de todos y ofreciendo nuevos canales de comunicación y de entretenimiento. Estas herramientas introducen nuevas vías de relación entre personas capaces de mejorar su calidad de vida" (Garrote et al., 2019, p. 100). Sin embargo, es importante tener en cuenta que el solo uso de las TIC como herramientas de soporte administrativo no garantiza la competitividad y la creación de valor, para lograrlo deben de ser utilizadas debidamente o ser integrada a una actividad primaria como es la mercadotecnia y articularlas en conjunto con las condiciones externas del entorno. Las TIC deben ser empleadas como medio para crear cadenas de valor en las organizaciones, de manera eficiente para definir y administrar procesos claves transformadores de recursos con un alto valor agregado (Ramírez et al., 2017).

La adopción de los sistemas informáticos es una tendencia actual para fortalecer las áreas de negocios, competitividad y gestión organizacional, ya que integran la información tanto de las actividades empresariales como de los usuarios o clientes, para establecer estrategias de marketing enfocados al mercado y al consumidor, además que tienen característica de flexibilidad, escalabilidad, adaptación, y expansión de uso a través de los medios de comunicación digitales. Es así que, en la actualidad, las modernas plataformas de sistemas informáticos se han convertido en la herramienta fundamental, indispensable y competitiva que sostienen la gestión de los procesos productivos y administrativos en las medianas y grandes empresas del sector público o privado, cuya fuente es la información almacenada en bases de datos y su principal objetivo es la automatización de los procesos para aumentar la productividad, desempeño y efectividad en toma de decisiones. Estos son los denominados Sistemas de Planificación de Recursos Empresariales (ERP, por sus siglas en ingles).

Los sistemas ERP fueron desarrollados a comienzos de 1990 por el Gartner Group's Computer-Integrated Manufacturing Service de Stanford (Oghazi et al., 2017), y son herramientas tecnológicas utilizadas para planificar los recursos disponibles de una organización, a través de la automatización de la información, cuyo objeto principal es la de satisfacer la demanda de las necesidades y servicios dentro de la gestión empresarial (González, 2004), esto permite a las organizaciones automatizar e integrar dentro de una base de datos toda la información de los procesos empresariales(Andonegui et al., 2005), además de compartir los datos y prácticas comunes, permitiendo el acceso a la información de manera más eficiente (Andonegui et al., 2010).

El uso del sistema ERP se ha identificado como un factor crítico para obtener el beneficio de una instalación ERP. Sin embargo, los antecedentes específicos del uso del ERP y su impacto en los beneficios del ERP siguen siendo en gran medida desconocidos (Abd et al., 2016). Estos autores, en su estudio, identificaron antecedentes del uso del sistema ERP y encuestaron a 157 usuarios finales de sistemas ERP en Estados Unidos. Los resultados que obtuvieron sugieren que el uso de sistemas ERP está directamente relacionado con los beneficios de estos sistemas. También pudieron identificar que la relación está determinada por el grado de mecanismos de integración del conocimiento dentro de la empresa para su gestión. En consonancia con su modelo, los resultados también revelaron que los recursos técnicos, el ajuste organizativo y el grado de implantación del ERP son factores clave para el uso de sistemas ERP (Abd et al., 2016). 
Los autores Ali y Miller (2017) en su estudio "ERP system implementation in large enterprises-a systematic literature review", concluyeron que el apoyo de la alta dirección, los buenos equipos de gestión de proyectos y las buenas comunicaciones son los tres factores críticos más importantes para el éxito de la implantación. Pero también identifican lagunas críticas en las investigaciones actuales sobre el tema, pues consideran que las mismas se centran predominantemente en la fase de implantación, pero faltan investigaciones sobre la fase previa y posterior a la implantación, y no se ha desarrollado ninguna metodología de implantación estándar en la industria (Ali \& Miller, 2017). No resulta casual el hecho de que la tendencia de las empresas e instituciones sea hacia incorporarse y acoplarse a la creación de una administración eficiente y eficaz, en donde se satisfaga las necesidades reales de los ciudadanos al menor coste posible, favoreciendo el desarrollo de servicios de mayor calidad, con sistemas de control que otorguen una plena transparencia de los procesos, planes y resultados, este es el paradigma de la nueva gestión pública.

Generalmente la percepción ciudadana respecto a la administración pública es lacerante, con cierto enfoque precario e incompetencia. Actualmente existe la convicción de un cambio de paradigma en las instituciones públicas, en donde es posible aplicar los conocimientos racionales y explícitos, habilidades, técnicas, enfoques, prácticas, estándares, o nuevos modelos de administración gerencial para resolver con eficacia los problemas sociales y mejorar los resultados de la acción que tomen las autoridades gubernamentales. El término nueva gestión pública (NPM) surge por los años 90 , el cual hace referencia a que la adopción y desarrollo de dinámicas, estándares, modelos de gestión y prácticas que se utilizan desde hace décadas en las empresas del sector privado, las mismas que puedan ser implementadas en los organismos del sector público y tener el mismo éxito que en el sector privado, con el objeto de responder con eficiencia y calidad a los servicios públicos, optimizando los recursos disponibles (Ebizor, 2016).

En Ecuador, el desarrollo e introducción de los sistemas ERP en sector gubernamental tiene una tendencia al crecimiento a través de proveedores nacionales e internacionales, debido a que la automatización de la información representa una permanente ventaja competitiva y de organización de procesos y productos con la aplicación de nuevas tendencias tecnológicas y acorde a las nuevas exigencias de calidad de servicio que responden los clientes y usuarios. Actualmente el gobierno nacional, a través del Ministerio de Telecomunicaciones y Sociedad de la Información (MINTEL), tiene la atribución de "Gestionar la política y directrices emitidas para la gestión de la implementación del gobierno electrónico; y, Desarrollar y coordinar planes, programas o proyectos sobre gobierno electrónico que sean necesarios para su implementación", función que estaba a cargo de Secretaría Nacional de Administración Pública. En este punto los gobiernos seccionales juegan un papel fundamental, ya que son los actores principales que impulsan la incorporación de las tecnologías de la información, las comunicaciones digitales y el uso del Internet, para responder con una gestión más eficaz de sus procesos, y bajo ese mismo enfoque empresarial, brindar los servicios públicos con calidad.

Por tanto es importante y casi imprescindible invertir en nuevas infraestructuras y plataformas tecnológicas con sistemas informáticos modernos que permitan gestionar y planificar todos los recursos de la organización, para satisfacer las demandas de los servicios ciudadanos, esto se encamina a una tendencia moderna de gestión pública denominada "gobierno electrónico", que en Ecuador es fomentada a través del Plan Nacional de Gobierno Electrónico 2018-2021 por el MINTEL, mismo que sigue las guías plasmadas en el Libro Blanco de la Sociedad de la Información 2018, que está basado en el Objetivo 7 del Plan Nacional de Desarrollo 2017-2021 cuya meta es mejorar el índice de gobierno electrónico a 2021.

En este ámbito, el Gobierno Autónomo Descentralizado (GAD), municipal del Cantón Riobamba, la inversión para la adquisición del sistema informático ERP con el nombre de SIIM 2 acrónimo de sistema informático integral multifinalitario, ha provocado una participación activa, integral antes y durante el proceso de implementación, que ha involucrado y comprometido a todas las áreas y niveles operativos que han conllevado a diversos cambios de procedimientos, instrumentos, personal capacitado, entre otros, lo cual no siempre ha sido favorable en el desarrollo normal del proceso de implementación.

\section{OTROS ANTECEDENTES}

Hasta el año 2015 el gobierno autónomo municipal de Riobamba, a través del soporte técnico del departamento de tecnologías, administraba la operatividad de varios sistemas informáticos, los cuales estaban desarrollados en distintos lenguajes de programación, almacenados en distintos motores de bases de datos e instalados en diferentes sistemas operativos como se muestra en la tabla 1. 
Tabla 1. Softwares y aplicaciones utilizados en el GAD Cantón Riobamba antes de la implementación del ERP

\begin{tabular}{|l|l|c|c|c|}
\hline $\begin{array}{l}\text { Nombre del } \\
\text { sistema }\end{array}$ & Módulos que contiene & $\begin{array}{c}\text { Sistema } \\
\text { Operativo }\end{array}$ & $\begin{array}{c}\text { Motor de Bases } \\
\text { de Datos }\end{array}$ & $\begin{array}{c}\text { Código } \\
\text { Fuente }\end{array}$ \\
\hline Cabildo & $\begin{array}{l}\text { Gestión de Catastros, Servicios Públicos, } \\
\text { determinación de impuestos y tasas, recaudación }\end{array}$ & Windows 2003 & Oracle & Disponible \\
\hline Sigame & $\begin{array}{l}\text { Gestión de contabilidad, tesorería, bodega, } \\
\text { activos fijos, recursos humanos }\end{array}$ & Windows NT & SQL & $\begin{array}{c}\text { No } \\
\text { disponible }\end{array}$ \\
\hline Bancos & Trasferencias, libros bancos, concilaciones & Windows 2003 & Foxpro & Disponible \\
\hline Roles & $\begin{array}{l}\text { Roles de pago para funcionarios a nombramiento } \\
\text { y contrato }\end{array}$ & Windows NT & Foxpro & Disponible \\
\hline Presupuesto & Presupuesto & Windows NT & Foxpro & Disponible \\
\hline $\begin{array}{l}\text { Sistema de } \\
\text { inventarios }\end{array}$ & Inventarios equipos informáticos & Windows 2003 & Mysql & Disponible \\
\hline Milenio & Contabilidad 2001 hasta 2006 & Windows NT & SQL & $\begin{array}{c}\text { No } \\
\text { disponible }\end{array}$ \\
\hline Finansga & Contabilidad 2007,2008,2009 & Windows NT & SQL & $\begin{array}{c}\text { No } \\
\text { disponible }\end{array}$ \\
\hline MegaFinan & Contabilidad 2010 & Windows NT & SQL & $\begin{array}{c}\text { No } \\
\text { disponible }\end{array}$ \\
\hline SRPR & Trámites del registro de la propiedad & Windows 2003 & SQL & $\begin{array}{c}\text { No } \\
\text { disponible }\end{array}$ \\
\hline
\end{tabular}

El hecho de administrar y controlar diferentes lenguajes de programación, distintas bases de datos, y diversos sistemas operativos para una misma entidad que tiene un mismo objetivo, provoca un desaprovechamiento de la capacidad tanto de los conocimientos de los profesionales técnicos de los sistemas informáticos, así como casos de duplicidad de datos, falta de integración entre sistemas por los diferentes formatos y la complicada posibilidad de compartir datos en línea y en tiempo real.

La transferencia de nuevas competencias, el incremento de procesos se incrementó significativamente en el GAD de Riobamba, debido a la magnitud y complejidad de sus procesos organizacionales, económicos, técnicos, patrimoniales, que aumentaron con la demanda de los servicios ciudadanos. Sin embargo, este GAD no contaba con una herramienta informática unificadora de la información en una plataforma única, que redujera las insuficiencias generadas por la duplicación de procesos, datos, variables, incompatibilidad de recursos informáticos que provocan incoherencia de información, desconfianza y complejidad de interpretación de los resultados obtenidos, además de dificultar una competitividad con otras instituciones acorde a las nuevas tendencias de servicios que se pueden brindar a los ciudadanos. Por otra parte, el anterior modelo de gestión implicaba pagos por suscripciones por licencias de software utilizadas, o por el número de equipos informáticos instalados, así como pagos por actualizaciones, nuevas versiones o requerimientos y a esto se suma la dependencia con los desarrolladores de los sistemas informáticos, ya que no se disponía de código fuente de los sistemas.

Con la implementación del sistema ERP para reducir los problemas antes mencionados en esta institución pública, la cual rinde cuentas a las instancias de control estatales; se hace necesario realizar una evaluación expost del proyecto, con el objetivo de identificar los resultados obtenidos, así como del grado de satisfacción que se generó al interior y exterior de la institución. Por otra parte, este es un proyecto importante para la gestión municipal desde el punto de vista financiero, ya que se trata de una inversión valorada en aproximadamente cuatrocientos cincuenta mil dólares (USD 450.000,00), por lo que, para los funcionarios a cargo de procesos gobernantes y administrativos, resulta medular la realización de una evaluación que permita inferir los resultados, efectos y percepción de la satisfacción de los beneficiarios de la nueva plataforma informática.

Es por ello que la presente investigación tuvo como objetivo realizar una evaluación expost al proyecto de implementación del Sistema Informático Integral Multifinalitario en el Gobierno Autónomo Descentralizado Municipal del Cantón Riobamba, para así verificar el cumplimiento de los objetivos planteados en el proyecto y evaluar el desempeño institucional y la calidad de los servicios municipales a partir de la implementación del sistema informático.

\section{METODOLOGÍA}

El estudio realizado es del tipo no experimental debido a que las variables o factores que se obtuvieron no son modificados, y la estrategia para el diseño de investigación es transversal puesto que las variables medidas representarán la respuesta a un fenómeno en un momento determinado, siendo este período entre el mes de octubre a noviembre del año 2020. El método científico principal aplicado fue el analítico y sintético en aras de, en concordancia con los datos e información recopilada, sustentar desde lo general, las 
particularidades de la presente investigación y arribar a las conclusiones específicas mediante el razonamiento lógico. De esta forma se pudieron evidenciar los efectos causados por la implementación del sistema ERP desde el conocimiento de la naturaleza del fenómeno objeto de estudio.

El enfoque de estudio fue cualitativo y cuantitativo con la finalidad de establecer de forma teórica cuales fueron los efectos tanto internos como externos, derivados de la implementación del sistema informático ERP. Se tomó como base el modelo de evaluación de Delone y McLean. Este modelo constituye una propuesta con amplio fundamento teórico sobre los aspectos importantes a mediar a la hora de evaluar un sistema de información, siendo además uno de los modelos ampliamente citados en la literatura revisada (Gable et al., 2003). Además, permite analizar y estudiar variables relacionadas al desarrollo de sistemas de información como son: la calidad del sistema, calidad de la información, calidad del servicio, uso y satisfacción, considerando que estas variables son una característica distintiva de la percepción del usuario en el uso de los sistemas de información, siendo uno de los métodos de amplia acogida en la literatura científica.

Acorde con el modelo de evaluación seleccionado, se definieron las dimensiones, indicadores y variables con el fin de determinar el cumplimiento de los objetivos planteados en el proyecto original de implementación del ERP. Para ello se utilizaron encuestas como instrumentos de medición, las cuales permitieron recopilar información de relevancia para el tema a través del método descriptivo, analizar y relacionar las variables para al final obtener un resultado. Asimismo, se obtuvo información a través de revisiones documentales y entrevistas, con el objetivo de contar con fuentes oficiales, varios aspectos relevantes que se evidenciaron luego de la implementación del sistema ERP. Las encuestas fueron dirigidas a los servidores municipales (usuarios internos) del GAD municipal de Riobamba, así también a los ciudadanos contribuyentes (usuarios externos), cuyo contenido consta de 51 variables, las cuales se identifican en 9 indicadores y articulados en 5 dimensiones.

Se identificaron las dimensiones: 1) Calidad del sistema, 2) Calidad de la información, 3) Calidad de servicio, 4) Uso y 5) Satisfacción del usuario. Así como se establecieron los indicadores: 1) Tiempo de respuesta, 2) Flexibilidad; 3) Facilidad de uso; 4) Confiabilidad; 5) Integridad; 6) Productividad; 7) Motivación de uso; 8) Efectividad en decisiones; y 9) Satisfacción de usuarios. La distribución de variables en las dimensiones seleccionadas se realizó en conformidad con la naturaleza propia de cada indicador y su relación con cada dimensión. Se utilizó como medición la escala de Likert de cinco puntos; 2 positivos, 2 negativos y 1 neutral, técnica que consiste en una escala ordenada y unidimensional para mediciones psicosométricas el cual evalúa actitudes. Es una técnica muy utilizada para medición de estudios de investigación en donde una serie de cuestionamientos son seleccionados con un grado de aceptación o rechazo por parte del participante de la encuesta.

Se aplicaron 5 cuestionarios con diversas preguntas con la finalidad de efectuar un levantamiento de información respecto a la cuantificación de las variables de estudio: 1) Cuestionario de encuesta para usuarios internos del sistema; 2) Cuestionario de encuesta para directores de gestión y líderes de subproceso; 3) Cuestionario de encuesta para administradores del sistema ERP; 4) Cuestionario de encuesta para ciudadanos contribuyentes; y 5) Cuestionario de entrevista semiestructurada para el director de gestión de tecnologías de la información. Las categorías utilizadas para las escalas de Likert aplicadas en cada uno de los 5 cuestionarios, se muestran en la tabla 2.

La población de estudio estableció en los funcionarios técnicos y administrativos que laboran en el GAD Municipal de Riobamba, quienes tiene acceso al sistema informático ERP y cuyas actividades diarias dependen del mismo. Esto comprende 14 direcciones de gestión, incluido 4 funcionalidades de gerencia y asesoría, cuya población total es de 425 usuarios. También formarán parte de la investigación los ciudadanos contribuyentes, ya que son los entes que hacen uso de los servicios que brinda la municipalidad, esto significa de acuerdo al sistema ERP un aproximado de 82535 contribuyentes.

Tabla 2. Escalas Likert aplicadas

\begin{tabular}{|c|l|l|l|l|l|}
\hline Valor Numérico & \multicolumn{5}{|c|}{ Categorías descriptivas utilizadas para la escala de Likert } \\
\hline 1 & Muy insatisfecho & Totalmente en desacuerdo & Nunca & Mínimo & Muy poco \\
\hline 2 & Insatisfecho & Parcialmente en desacuerdo & Casi nunca & Poco & Poco \\
\hline 3 & Neutral & Neutral & A veces & Nada & Neutral \\
\hline 4 & Satisfecho & Parcialmente de acuerdo & Frecuentemente & Mucho & Moderado \\
\hline 5 & Muy Satisfecho & Totalmente de acuerdo & Diariamente & Total & Bastante \\
\hline
\end{tabular}


Para realizar los análisis individuales y comparados de las dimensiones, se calcularon las medias ponderadas para cada indicador en base a las evaluaciones obtenidas, para obtener una evaluación media agregada ponderada para cada dimensión. La muestra empleada para ésta investigación consta de un muestreo estratificado proporcional aleatorio, formado por los datos obtenidos en las direcciones de gestión del GAD municipal tal como se ilustra (para el caso de los servidores municipales) en la tabla 3 conforme al total de la población objetivo, considerando un $95 \%$ de confiabilidad y un error del $5 \%$.

Tabla 3: Población de servidores municipales por cada dirección de gestión.

\begin{tabular}{|l|c|c|}
\hline Estrato & Población & Porcentaje \\
\hline Dirección de Gestión Financiera & 51 & $12 \%$ \\
\hline Dirección de Gestión Administrativa & 34 & $8 \%$ \\
\hline Dirección de Gestión de Talento Humano & 24 & $6 \%$ \\
\hline Dirección de Gestión de Servicios Municipales & 19 & $4 \%$ \\
\hline Dirección de Gestión de Ordenamiento Territorial & 70 & $16 \%$ \\
\hline Dirección de Gestión de Obras Públicas & 11 & $3 \%$ \\
\hline Dirección de Gestión Ambiental, Salubridad e Higiene & 14 & $3 \%$ \\
\hline Dirección de Gestión de Patrimonio & 8 & $2 \%$ \\
\hline Registro de la Propiedad & 39 & $9 \%$ \\
\hline Dirección de Gestión de Movilidad, Tránsito y Transporte & 23 & $5 \%$ \\
\hline Directores Departamentales & 20 & $5 \%$ \\
\hline Líderes de equipo & 50 & $12 \%$ \\
\hline Secretarías & 42 & $10 \%$ \\
\hline Asesores y Asistentes & 20 & $5 \%$ \\
\hline Total & 425 & $100 \%$ \\
\hline
\end{tabular}

Se aplicó la fórmula para el cálculo de tamaño de muestra para población finita y conocida, y se obtuvo un tamaño de muestra de 202 usuarios internos y 382 usuarios externos. Para comprobar la fiabilidad, consistencia interna y validez del constructo, se utilizó el método estadístico de Alfa de Cronbach y un análisis factorial por cada grupo de unidad de estudio mediante la Medida Kaiser-Meyer-Olkin y la Prueba de esfericidad de Bartleet (Dowdy et al., 2011). Para ampliar el tema de investigación acerca de los efectos e impacto institucional después de la implementación del ERP, se aplicó una entrevista semiestructurada dirigida al responsable del proyecto, en este caso corresponde al Director de Gestión de Tecnologías de la Información del GAD Municipal, quién es la persona que conoce a profundidad del tema y es la fuente primaria de información, y quién dirige al cuerpo técnico de ingenieros que son los responsables de la funcionalidad de todos los módulos informáticos del ERP.

Por último, se procuró demostrar una relación estadísticamente significativa entre las dimensiones Calidad y Satisfacción del usuario, mediante la aplicación de las pruebas Chi-cuadrado de Pearson y de Verosimilitud. Para esto se confeccionó una tabla de contingencia o doble entrada, que resume simultáneamente las dos variables de interés a partir de las frecuencias absolutas observadas en cada categoría cruzada. Las herramientas informáticas empleadas para el procesamiento de la información fueron, el software Microsoft Excel y el paquete estadístico IBM SPSS en su versión 23, que son utilizados en la investigación para las áreas de las ciencias sociales y económicas. Estos sistemas informáticos son las herramientas informáticas que facilitan el análisis estadístico descriptivo, análisis de índices de fiabilidad del constructo, análisis factorial de variables, entre otros análisis desarrollados en esta investigación.

\section{RESULTADOS Y DISCUSIÓN}

Inicialmente se procedió a medir la fiabilidad de las encuestas aplicadas, cuyos resultados se detallan a continuación para cada uno de los estratos identificados. Para las variables aplicadas a los servidores municipales, se obtuvo un Alfa de Cronbach global de 0.931, lo cual indica una alta consistencia interna, y esto se corroboró en el análisis individual de cada variable, con un recorrido del estadístico de Cronbach, entre 0.922 y 0.937 , lo que evidencia la pertinencia de cada una de las variables evaluadas. Algo similar se evidenció en los otros estratos encuestados. Para el caso de directores de gestión y líderes de subprocesos, se obtuvo un Alfa de Cronbach general de 0.896 con un recorrido individual entre 0.895 y 0.912 y para el estrato de los contribuyentes los resultados fueron de 0.854 para el estadístico y un valor mínimo individual de 0.806 y un máximo de 0.875 . Para profundizar la comprobación de la validez de los instrumentos de investigación se procedió a aplicar la Medida Kaiser-Meyer-Olkin (KMO) y la Prueba de esfericidad de Bartleet por cada grupo de unidad de estudio. Los valores del KMO obtenidos fueron superiores a 0.86 en todos los casos, y la significatividad de la prueba Chi-cuadrado aplicada (para un nivel de confianza del 95\%) fue de 0 , para cada uno de los estratos. Estos resultados confirman la alta consistencia de los instrumentos aplicados. 
Una vez demostrada la factibilidad de las encuestas aplicadas, se procesó la información estadística recopilada para cada dimensión según los indicadores. Se identificó primeramente que la media estadística correspondiente a la antigüedad laboral de los servidores públicos del GAD Municipal es de aproximadamente 7 años, mientras que para el grado de conocimiento en el uso de equipos y sistemas informáticos evaluado en las categorías; básico, intermedio y avanzado, se obtuvieron las frecuencias relativas $0.077,0.827$ y 0.096 respectivamente. Estos resultados denotan una experiencia laboral significativa, así como un adecuado nivel de conocimiento en el uso de sistemas informáticos, lo cual ofrece una mayor fiabilidad de la información aportada por los encuestados.

Por otra parte, se midió el grado de conocimiento de varios factores por parte de los contribuyentes, como es el caso de qué tanto están informados acerca de las nuevas alternativas de acceso a pagos de servicios y consultas que pueden hacer en la municipalidad. Así se pudo conocer que un $52.10 \%$ de contribuyentes no tienen conocimiento de las alternativas de consulta a través del servicio de internet o aplicación móvil, o de servicios para pagos en diferentes agencias municipales y entidades bancarias. Para cuantificar la dimensión Calidad del sistema informático, se utilizaron los indicadores: tiempo de respuesta, la flexibilidad y facilidad de uso. La evaluación media agregada ponderada de la dimensión para los Servidores públicos y los Contribuyentes fue de 4.15 y 3.12 respectivamente, lo que indica una peor percepción de la Calidad del sistema por parte de estos últimos. Al consolidar las frecuencias relativas de los resultados de las encuestas respecto a esto tres indicadores, tanto para los servidores públicos como para los contribuyentes, se pudo conformar el histograma comparativo de frecuencias relativas consolidadas que se muestra en la figura 1.

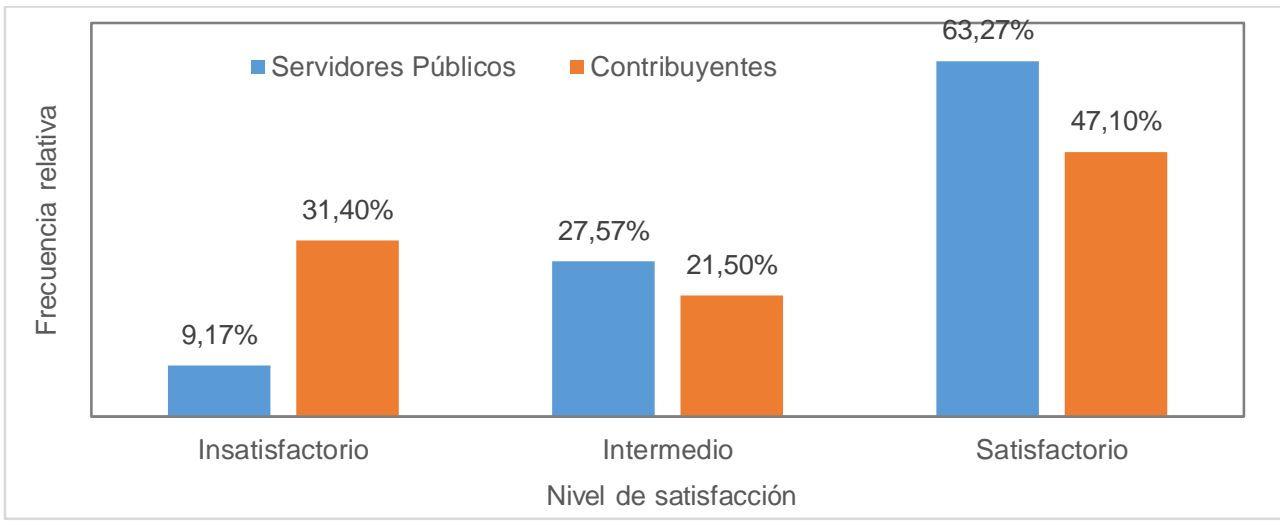

Figura 1. Percepción de la calidad del sistema informático

Se resumió la escala Likert utilizada en tres nuevas categorías y se puede advertir una notable diferencia del grado de insatisfacción entre los servidores municipales (9.17\%) y de los contribuyentes (31.4\%), destaca además que solo el $47.1 \%$ de estos últimos, tiene un criterio positivo para la dimensión evaluada. Por tanto, existe una opinión negativa respecto a la calidad del sistema por parte de los contribuyentes, mientras que el indicador más significativo en este comportamiento es el relativo a los tiempos de respuesta con casi un $40 \%$ de insatisfacción para el estrato, mientras q los servidores públicos muestran un alto nivel de satisfacción general. Se debe mejorar la consecución de los procesos que se llevan dentro de la institución por parte de los servidores municipales y de la supervisión de los productos entregados por parte de los directores de gestión y líderes de procesos como parte administrativa y operativa de la institución.

La dimensión Calidad de la información fue valorada mediante el uso de los indicadores: confiabilidad, integridad y precisión. En la tabla 4 se muestran las evaluaciones medias ponderadas agregadas y las frecuencias relativas generales de la dimensión para cada uno de los estratos encuestados. Una vez más son los contribuyentes los que muestran un menor nivel de satisfacción, con una media ponderada de 3.04 y una frecuencia relativa de $19.1 \%$, mientras que los directivos presentan el mayor porciento de satisfacción general respecto a la calidad de la información, con 85.3 y una evaluación media de 4.26.

Tabla 4. Evaluaciones medias y frecuencias relativas de la Calidad de la información

\begin{tabular}{|l|l|l|l|l|}
\hline Estratos & Evaluación Media & Insatisfactorio & Intermedio & Satisfactorio \\
\hline Servidores Públicos & 3,42 & $12,5 \%$ & $22,3 \%$ & $65,2 \%$ \\
\hline Contribuyentes & 3,04 & $19,1 \%$ & $22,0 \%$ & $58,9 \%$ \\
\hline Directivos & 4,26 & $0,0 \%$ & $14,7 \%$ & $85,3 \%$ \\
\hline
\end{tabular}


Destaca en este caso que los contribuyentes tienden a estar insatisfechos a una escala mayor que los servidores públicos, esto refleja que se debe orientar el mejoramiento de los procedimientos y procesos en favor de los clientes externos, fortalecer el servicio a partir de la mejora de la Calidad de la información que el sistema informático provee a los ciudadanos.

Para la dimensión Uso, se consideró el indicador Motivación del uso del sistema, mediante el cual los clientes internos (Servidores públicos y Directivos), en calidad de usuarios, evaluaron su disposición para utilizar el sistema en el desarrollo de sus actividades laborales diarias. Estos estratos mostraron evaluaciones medias respectivas de 3.95 y 3.11. Se verificó de esta forma que, aun cuando en ambos estratos la frecuencia de opiniones negativas es considerablemente baja (inferior al $8 \%$ en ambos), los Directivos denotaron una mayor frecuencia con de más del $73 \%$ de los encuestados con un nivel medio de uso del sistema, mientras que los Servidores públicos mostraron una alta motivación al uso frecuente con un $65 \%$. Por tanto, es necesario incrementar la motivación de parte de los directivos hacia la utilización del sistema en aras de fortalecer la utilidad del sistema implementado a todos los niveles dentro del GAD Municipal.

La Calidad del servicio está orientada como dimensión para medir la percepción global del usuario acerca de manera en que los servicios son proporcionados y proveídos, a pesar de que son subjetivos, permiten enfocar la satisfacción en cuanto al cumplimiento de sus necesidades. Par tal fin se utilizaron los indicadores: productividad, efectividad para la toma de decisiones por parte de los Servidores públicos, empatía percibida por parte de los contribuyentes. El valor medio de la evaluación por estratos para los Contribuyentes fue de 2.98, mientras que el de los Servidores públicos fue superior en 1.24 puntos. En la figura 2 se muestran las frecuencias relativas globales para la evaluación de esta dimensión.

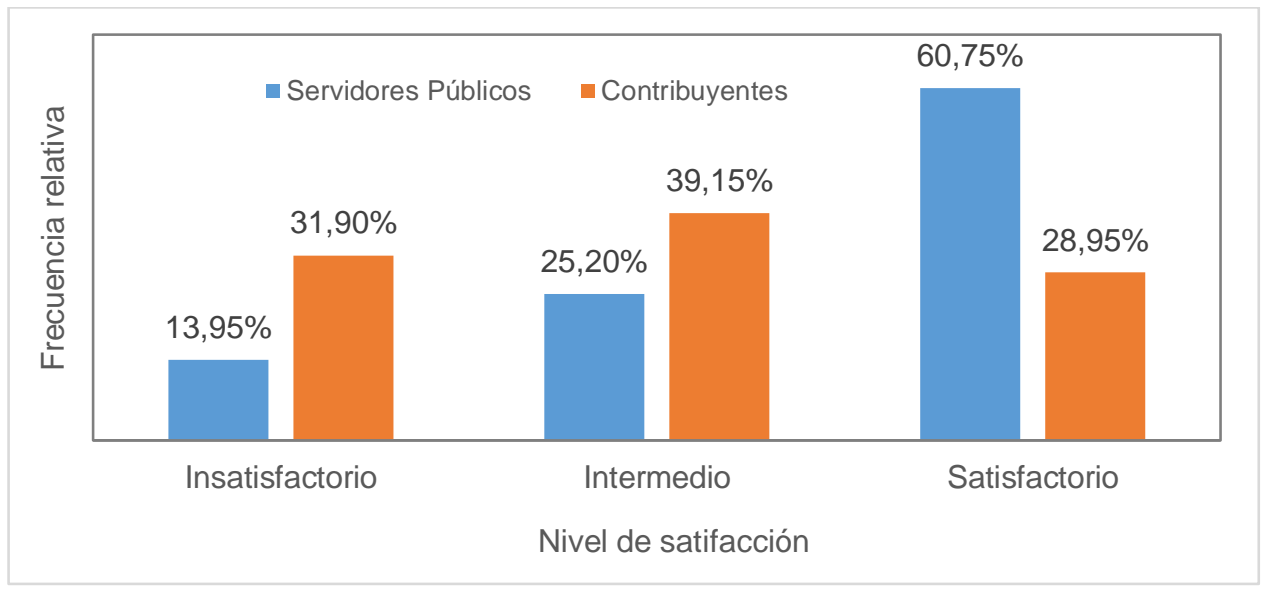

Figura 2. Percepción general de la calidad del servicio

Los resultados de la dimensión de calidad de servicio satisfactoria en un $60.75 \%$ para los Servidores públicos institucionales, existiendo una notable diferencia de apreciación por parte de los contribuyentes con apenas un $28.95 \%$ de satisfacción, representa una desfavorable percepción, ya que el $31.90 \%$ está insatisfecho y el $39.15 \%$ es indiferente al cambio. Estos resultados permiten comprobar que los ciudadanos no perciben una mejoría en la calidad de los servicios institucionales, a pesar de que los servidores municipales cuentan con la herramienta informática que facilita y ofrece ventajas al interior de las labores cotidianas dentro de la institución. Se debe tener en cuenta por parte de los responsables de recursos humanos y directores departamentales para mejorar estos indicadores.

Para determinar la Satisfacción de usuario después de la implementación del sistema informático ERP, se usaron los indicadores: soporte técnico, capacitación, conocimiento y acceso a los servicios. En la tabla 5 se exponen los valores de la evaluación media ponderada agregada y las frecuencias relativas generales de la dimensión para cada uno de los estratos.

Tabla 5. Evaluaciones medias y frecuencias relativas de la Satisfacción de usuario

\begin{tabular}{|l|l|l|l|l|}
\hline Estratos & Evaluación Media & Insatisfactorio & Intermedio & Satisfactorio \\
\hline Servidores Públicos & 3,68 & $16,1 \%$ & $24,1 \%$ & $59,9 \%$ \\
\hline Contribuyentes & 3,1 & $31,8 \%$ & $39,3 \%$ & $28,9 \%$ \\
\hline Directivos & 4,14 & $2,2 \%$ & $25,3 \%$ & $72,5 \%$ \\
\hline
\end{tabular}


Una vez más se identifica una menor satisfacción por parte de los Contribuyentes, con una evaluación media de solo 3.1 y una frecuencia relativa de insatisfacción de un $31.8 \%$, a esto se suma como resultado negativo, un alto nivel de neutralidad para este estrato con un 39.3\%. En contraposición a esto, solo el $2.2 \%$ de los Directivos opinó negativamente y un $72.5 \%$ tiene un criterio positivo como usuario del sistema. Al término de esta investigación se procedió a verificar estadísticamente si existe una relación significativa entre las dimensiones de calidad medidas en la evaluación del sistema informático ERP implementado en el Gobierno Autónomo Descentralizado del Cantón Riobamba y el grado de satisfacción de los usuarios con los servicios municipales. Los resultados de las pruebas Chi-cuadrado de Pearson y Verosimilitud realizadas para cada estrato arrojaron una significación asintótica igual a cero. Ya que el nivel de confianza propuesto en la investigación es del 95\%, se comprueba en ambos casos (estratos), que existe una relación directa entre las dimensiones estudiadas, por lo tanto, se acepta la hipótesis de investigación planteada para este análisis, en donde la calidad tiene una relación directa con la satisfacción.

\section{CONCLUSIONES}

A partir de la evaluación de los indicadores y dimensiones estudiadas del sistema informático ERP implementado en el gobierno autónomo descentralizado municipal del Cantón Riobamba, los resultados obtenidos permiten concluir que: 1) el sistema ofrece varios beneficios relacionados con la gestión organizacional, como la centralización, unificación e integración de la información, confiabilidad y precisión de los datos, principalmente para los Servidores públicos, que resultó además el grupo más lo utiliza; 2) los Directivos, mostraron el mayor nivel de satisfacción como usuarios del sistema para la gestión de los procesos; 3) a pesar de las innegables ventajas del sistema para el desempeño laboral de los clientes internos, se constató una percepción desfavorable del mejoramiento de la calidad del servicio y la satisfacción a los ciudadanos, siendo necesario mejorar éstos factores de atención por parte de los Servidores municipales y los Directivos relacionados con la implementación y mejora continua del ERP; 4) se demostró estadísticamente la relación directa existente entre las dimensiones Calidad y satisfacción del usuario en la muestra estudiada.

\section{REFERENCIAS}

Abd Elmonem, M. A., Nasr, E. S., y Geith, M. H., Benefits and challenges of cloud ERP systems-A systematic literature review. Future Computing and Informatics Journal, 1(1-2), 1-9 (2016).

Ali, M., y Miller, L., ERP system implementation in large enterprises-a systematic literature review, Journal of Enterprise Information Management, (2017).

Andonegi Martínez, J. M., Fa, M. C., y Elguezabal, I. Z. E., Evolución histórica de los sistemas ERP: de la gestión de materiales a la empresa digital, 12, 61-72 (2005).

Andonegui, M., Casadesus, M., y Zamanillo, I., Evolución histórica de los Sistemas ERP de la gestión de materiales a la empresa digital, Revista de Dirección y Administración de empresas, 1-19 (2010).

Ebizor, F. La Nueva Gestión Pública. Recuperado 6 de agosto de 2018, de http://www.ebizor.com (2016).

Delone, W. H., y McLean, E. R., The DeLone and McLean Model of Information Systems Success: A Ten-Year Update, https://doi.org/10.1080/07421222.2003.11045748, Journal of Management Information Systems, 19(4), 9-30, (2003).

Dowdy, S., Wearden, S., y Chilko, D., Statistics for research (Vol. 512), John Wiley y Sons, (2011).

Garrote, R. D, Jiménez, F. S, y Gómez, B. I., La Gestión por Procesos en las Pymes de Barranquilla: Factor Diferenciador de la Competitividad Organizacional, http://dx.doi.org/10.4067/S0718-07642019000200103, Información Tecnológica, 30(2), 103-114 (2019).

Haddara, M., y Elragal, A., The Readiness of ERP Systems for the Factory of the Future, Procedia Computer Science, 64, 721-728 (2015).

Huitema, B., The Analysis of Covariance and Alternatives: Statistical Methods for Experiments, Quasi-Experiments and Single-Case Studies, 2nd Edition, Wiley, New Jersey, USA (2011)

Korolov, M., El futuro del ERP es la Inteligencia Artificial. Recuperado 31 de julio de 2018, de https://cioperu.pe (2018).

Oghazi. P, Rad, F. P, Karlsson. S, y Haftor. D., RFID and ERP systems in supply chain management,

http://dx.doi.org/10.1108/EJMBE-02-2018-0031, European J. Manag. and Business Economics, 27(2), 171-182 (2018).

Ramírez, M., Arellano, A., y Carballo, B. Modelo conceptual de gestión organizacional como referente en el desarrollo de un sistema de información de mercadotecnia integral. http://dx.doi.org/10.17993/3ctic.2017.58.54-693C, TIC:

Cuadernos de desarrollo aplicados a las TIC, 6(4), 54-69. (2017).

Rajan, C. A., y Baral, R., Adoption of ERP system: An empirical study of factors influencing the usage of ERP and its impact on end user, IIMB Management Review, 27(2), 105-117 (2015).

Tamayo, G.P., Metodología para la integración de los sistemas de gestión organizacional, Ciencias Holguín, XXI (3), 118 (2015). 
